\title{
EVAPOTRANSPIRAÇÃO POTENCIAL E COEFICIENTE DA CULTURA DE DOIS GENÓTIPOS DE ARROZ DE TERRAS ALTAS
}

\author{
Gentil Cavalheiro Adorian ${ }^{1}$, Rogério Lorençoni ${ }^{2}$, Durval Dourado Neto ${ }^{1}$, Klaus Reichardt ${ }^{3}$
}

\footnotetext{
${ }^{1}$ Universidade de São Paulo - Escola Superior de Agricultura Luiz de Queiroz, E-mail: gentil.cav@gmail.com, ddourado@usp.br,

${ }^{2}$ Universidade Federal do Tocantins, E-mail: lorenconi@uft.edu.br

${ }^{3}$ Universidade de São Paulo - Centro de Energia Nuclear na Agricultura, E-mail: klaus@cena.usp.br
}

\section{RESUMO}

Objetivou-se com este estudo avaliar a evapotranspiração potencial e calcular o coeficiente da cultura de dois genótipos de arroz de terras altas, moderno e tradicional, numa tentativa de verificar diferenças na tolerância à seca. O genótipo de arroz de terras altas Batatais apresentou maior evapotranspiração, na $8^{\mathrm{a}}$ semana após o plantio, que o genótipo BRSPrimavera. Conforme a relação do coeficiente da cultura e o índice de área foliar estimado, o genótipo BRS-Primavera apresentou maior evapotranspiração por índice de área foliar.

Palavras-chave: Estresse hídrico, evapotranspiração, Oryza sativa

\section{POTENTIAL EVAPOTRANSPIRATION AND CROP COEFFICIENT OF TWO UPLAND RICE GENOTYPES}

\section{ABSTRACT}

This study evaluates the potential evapotranspiration and calculates the crop coefficient of two highland rice genotypes, modern and traditional, in order to verify differences in relation to drought tolerance. The rice genotype highland Batatais showed evapotranspiration, on the 8th week after planting, higher than the BRS-Primavera genotype. According to the relation of crop coefficient and the estimated leaf area index the BRS-Primavera showed higher evapotranspiration by leaf area index.

Keywords: Hydric stress, evapotranspiration, Oryza sativa

\section{INTRODUÇÃO}

O arroz (Oryza sativa L.) é cultivado em vários locais do planeta, sendo China, Índia, Indonésia, Vietnã, Tailândia,
Blangadesh, Myamar, Filipinas, Brasil e Japão os maiores produtores. No Brasil, o arroz é o terceiro cereal mais produzido, atrás somente da soja e do milho e teve, em 
2013, produção total de 11.758 .873 toneladas (IBGE, 2014).

O cultivo do arroz sofre sérias restrições socioambientais em seu sistema irrigado (ANA, 2009). Sendo assim, é necessária a ampliação do cultivo de arroz em sistema de terras altas para manter ou mesmo melhorar o patamar de produção do arroz, com redução do gasto de água. A região de Cerrados no Brasil é a mais importante no cultivo tradicional de arroz de terras altas (FORNASIERI FILHO \& FORNASIERI, 2006; GUIMARÃES et al., 2006). Porém, nessa região, ocorrem períodos de estresse por falta de chuvas regulares, intervalos conhecidos como "veranicos", em frequência e duração distintas (DATTA, 1981). Nas plantas, esses períodos secos suficientemente prolongados promovem estresse hídrico, que limita o crescimento e o desempenho de plantas cultivadas mais do que qualquer outro fator socioambiental, ocasionando maior perda de produtividade, no caso do arroz, no período reprodutivo (HEINEMANN \& STONE, 2009).

$$
\text { O conhecimento }
$$

da evapotranspiração potencial da cultura, em condições ótimas de disponibilidade de água no solo, juntamente com a evapotranspiração de referência, que é a perda de água por unidade de tempo e de área de uma cultura padrão, permitem calcular o coeficiente da cultura $(\mathrm{Kc})$, que indica o quanto a cultura de interesse necessita de água a mais que a cultura de referência. Tal coeficiente é extremamente útil no manejo da irrigação.

No cultivo do arroz, a determinação do Kc é geralmente feita sem distinção de cultivares, levando a padronização do Kc e considerando apenas a cultura e não o genótipo. Entretanto, os valores de evapotranspiração podem variar entre cultivares, o que demonstra a necessidade de conhecimento do Kc para cada genótipo.

Assim, o presente estudo teve como objetivo avaliar a evapotranspiração potencial e calcular o coeficiente da cultura de dois genótipos de arroz de terras altas, um caracterizado como moderno e outro como tradicional, numa tentativa de verificar diferenças na tolerância à seca.

\section{MATERIAL E MÉTODOS}

O trabalho foi conduzido no ano de 2013, em casa de vegetação, na Escola Superior de Agricultura "Luiz de Queiroz" ESALQ/USP, localizada no município de Piracicaba - SP, à $22^{\circ} 43^{\prime}$ de latitude sul e $47^{\circ} 38^{\prime}$ de longitude oeste, e altitude aproximada de 547 metros. O clima do 
município é classificado como subtropical úmido com estiagem no verão (Cwa).

Para o experimento foram utilizados dois genótipos de arroz de terras altas: 'Batatais', arroz tradicional, e 'BRSPrimavera', arroz moderno. $\mathrm{O}$ arroz 'Batatais' foi semeado no dia 21 e o arroz 'BRS-Primavera' no dia 23 de dezembro de 2012, em bandejas de isopor contendo substrato agrícola comercial, em vasos contendo $9 \mathrm{~kg}$ de solo seco, com volume de $6.221 \mathrm{~cm}^{3}$ e área superficial de $615,7 \mathrm{~cm}^{2}$. Os genótipos foram semeados em datas diferentes para que germinassem em data aproximada e tivessem tamanho semelhante na época do transplante.

Para adubação de plantio foi utilizado 2,5 g.vaso ${ }^{-1}$ do formulado NPK 28-08-16 + 2 g.vaso $^{-1}$ de Yoorin $^{\circledR}$. Para o controle de pragas foi utilizado Deltametrina 25CE, na dosagem de $40 \mathrm{ml}$ para $100 \mathrm{~L}$ de água, aplicados em momento de ataque de lagartas. O controle de plantas daninhas foi feito manualmente à medida que as mesmas foram surgindo.

Para avaliar a evapotranspiração da cultura, doze vasos delineados em blocos casualizados foram utilizados, sendo dois genótipos e seis repetições. Durante todo período de condução do experimento os vasos foram pesados diariamente pela manhã e quantificada a quantidade de água perdida para a atmosfera, pela diferença do peso na capacidade de vaso e o peso constatado no dia. A diferença de água convertida em volume $(1 \mathrm{~g}$ de água $=1 \mathrm{ml})$ dividida pela área da superfície do solo no vaso resultou na evapotranspiração da cultura em milímetros. Os valores de ETc foram corrigidos, descontando o peso diário das plantas, os quais foram estimados, a partir dos pesos reais dos vasos cultivados paralelos, constatados nos estádios (R2, R5 e R9). Dos dados de ETc diários corrigidos do peso das plantas, foram feitas médias semanais, para que um valor de ETc $\left(\mathrm{mm} \cdot \mathrm{dia}^{-1}\right)$ representasse cada semana, de forma a reduzir as variações e simplificar as análises. Portanto, no total do ciclo dos genótipos foram determinados doze valores de ETc, um para cada semana.

A evapotranspiração de referência foi estimada utilizando dois métodos: Thornthwaite (1948) e Hargreaves \& Samani (1985). Os resultados são em mm.dia ${ }^{-1}$ e foi utilizado um valor médio de $\mathrm{ET}_{0}$ diário para representar uma semana. A partir dos valores médios obtidos da evapotranspiração da cultura (ETc, mm.dia ${ }^{-1}$ ) e a partir dos valores estimados da evapotranspiração de referência $\left(\mathrm{ET}_{0}, \mathrm{~mm} \cdot \mathrm{dia}^{-1}\right)$, foi possível determinar o coeficiente da cultura (Kc). 
Foram calculados dois coeficientes da cultura para cada genótipo, um para cada método de estimativa da $\mathrm{ET}_{0}$ utilizado.

Seis plantas de cada genótipo foram cultivadas separadamente para obter dados de índice de área foliar (IAF), coletadas em três estádios de desenvolvimento da cultura (R2, R5 e R9). Dessas, determinou-se primeiramente a área foliar das plantas de arroz, usando o método dos discos foliares. A área foliar foi calculada através de equação. Posteriormente, com a relação entre a área foliar e a área da superfície do solo do vaso, calculou-se o índice de área foliar (IAF), para ter conhecimento da área total das folhas em relação à área do solo do vaso que as mesmas estavam cobrindo. Os valores de IAF calculados são dependentes da área superficial do vaso que, não necessariamente, representa a área em que uma planta de arroz ocuparia no campo. Portanto, para qualquer alteração na área do vaso, tem-se alteração nos valores de IAF, o que impossibilita a comparação com IAF obtidos em campos. Porém, no atual experimento, tornou-se útil para comparar os genótipos avaliados.

Com o propósito de obter valores de índice de área foliar em cada dia do ciclo dos genótipos, os valores de IAF foram estimados a partir dos valores reais coletados dos três estádios referidos (R2, R5 e R9), para todos os dias do ciclo da cultura, determinando o primeiro dia (dia do transplante) como o dia 0 (zero) com IAF $=$ 0 (zero), e supondo que o crescimento do IAF do dia 0 até a primeira data de coleta (estádio R2) ocorreu de forma linear, e de R2 até R5, e de R5 até R9, também ocorreu de forma linear, foi possível estimar o índice de área foliar (IAFest), através da equação IAFest $=\mathrm{IAF}_{1}+\left[\left(\mathrm{IAF}_{2}-\mathrm{IAF}_{1}\right) /\left(\mathrm{D}_{2}-\mathrm{D}_{1}\right)\right] *(\mathrm{D}-$ $\left.\mathrm{D}_{1}\right)$, onde, $\mathrm{IAF}_{1}=$ índice de área foliar inicial; $\mathrm{IAF}_{2}=$ índice de área foliar final; $\mathrm{D}_{1}$ $=$ dia inicial; $\mathrm{D}_{2}=$ dia final; e $\mathrm{d}=$ dia desejado do ciclo para o referido IAFest.

Os valores médios semanais do índice de área foliar estimados (IAFest) de cada genótipo foram relacionados em função dos valores médios do coeficiente da cultura calculados $\left(\mathrm{K}_{\mathrm{cl}}\right.$, utilizando $\mathrm{ET}_{0}$ estimada pelo método de Thornthwaite e $\mathrm{Kc}_{2}$, utilizando $\mathrm{ET}_{0}$ estimada pelo método de Hargreaves \& Samani). Os dados de evapotranspiração potencial da cultura, coeficiente da cultura e coeficiente da cultura em função do índice de área foliar foram submetidos à análise de regressão.

\section{RESULTADOS E DISCUSSÃO}

Foram observados maiores valores de ETc para o arroz Batatais, diferindo do arroz 
BRS-Primavera, na $8^{\mathrm{a}}$ semana após o transplante (SAT) (Figura 1). A maior evapotranspiração de 'Batatais' pode estar relacionada com seu maior IAF, pois, em ambientes com condições sem limitação hídrica; quanto maior a área foliar, maior é a superfície transpirante da planta e maior é a perda de água (DUCHEMIN et al., 2006).

O ponto máximo de evapotranspiração (ETc) foi na nona semana após o transplante (SAT), com valores médios semanais de $10 \mathrm{~mm}^{-d_{i a}{ }^{-1}}$ para 'Batatais' e 9,2 mm.dia' ${ }^{-1}$ para 'BRSPrimavera' (Figura 1). Chan \& Cheong (2001) observaram evapotranspiração de até 12,4 mm.dia ${ }^{-1}$ em plantas de arroz cultivadas em calhas plásticas dentro de estufa.

O modelo de regressão que melhor se adequou aos dados de ETc foi o polinomial de terceiro grau, com $\mathrm{R}^{2}$ de $88,72 \%$ para 'Batatais' e 86,29\% para 'BRS Primavera', demonstrando leve decréscimo da $1^{\mathrm{a}}$ até a $2^{\mathrm{a}}$ semana após o transplante (SAT), aumento da $2^{\mathrm{a}}$ até a $9^{\mathrm{a}}$ SAT e a partir dessa, decréscimo até o final do ciclo da cultura. $\mathrm{O}$ maior valor de ETc da $1^{\text {a }}$ SAT em comparação à $2^{\mathrm{a}} \mathrm{SAT}$ pode ser resultado da evaporação do solo por causa da maior exposição da sua superfície ao sol (LILLEY $\&$ FUKAI, 1994), que na $2^{\mathrm{a}} \mathrm{SAT}$ apresentava-se mais sombreada devido ao desenvolvimento da planta. A elevação da evapotranspiração da cultura a partir da $2^{\mathrm{a}}$ SAT é devida ao desenvolvimento da planta e seu consequente acréscimo de índice de área foliar (IAF), que aumenta o consumo de água disponível no solo do vaso (ABDULLAHI et al., 2013).

Os valores estimados de Evapotranspiração de Referência $\left(\mathrm{ET}_{0}\right)$ apresentaram oscilações ao longo do tempo (Figura 2), que seguem as variações da temperatura. Os valores mais altos de $\mathrm{ET}_{0}$ foram observados para o método de Hargreaves \& Samani (1985), com médias variando de 4,7 a 7,5 mm.dia ${ }^{-1}$. O método de Thorthwaite (1948) apresentou médias variando de 3,7 a 5,7 mm.dia ${ }^{-1}$. Praveen et al. (2012) comparando vários métodos de estimativa da evapotranspiração de referência, também observaram maiores valores para o método de Hargreaves e Samani, quando comparado com os métodos de Priestly-Taylor, Penmam-Monteith, Kimberly-Penmam, e outros. Lu et al. (2005) registraram maiores valores de $\mathrm{ET}_{0}$ para o método de Hargreaves \& Samani e os menores valores para o método de Thornthwaite na região sudeste dos Estados Unidos, porém, o método de Thornthwaite melhor se correlacionou com a evapotranspiração. 


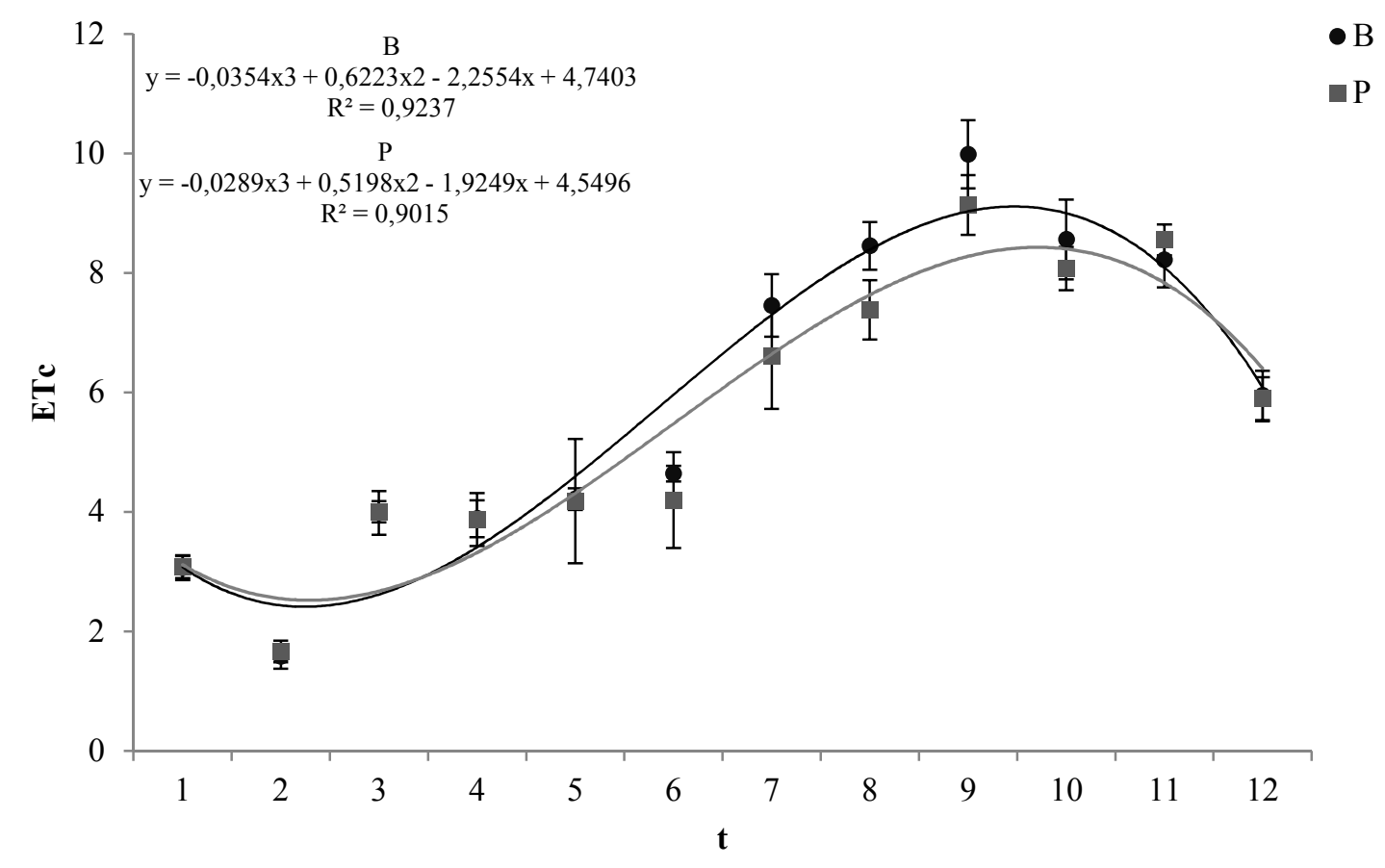

Figura 1. Valores de médias semanais de evapotranspiração da cultura (ETc, mm.dia ${ }^{-1}$ ) de cultivares de arroz de terras altas, 'Batatais' (B) e 'BRS Primavera' (P), em função do tempo (t, semanas após o transplante). Piracicaba-SP, 2013.

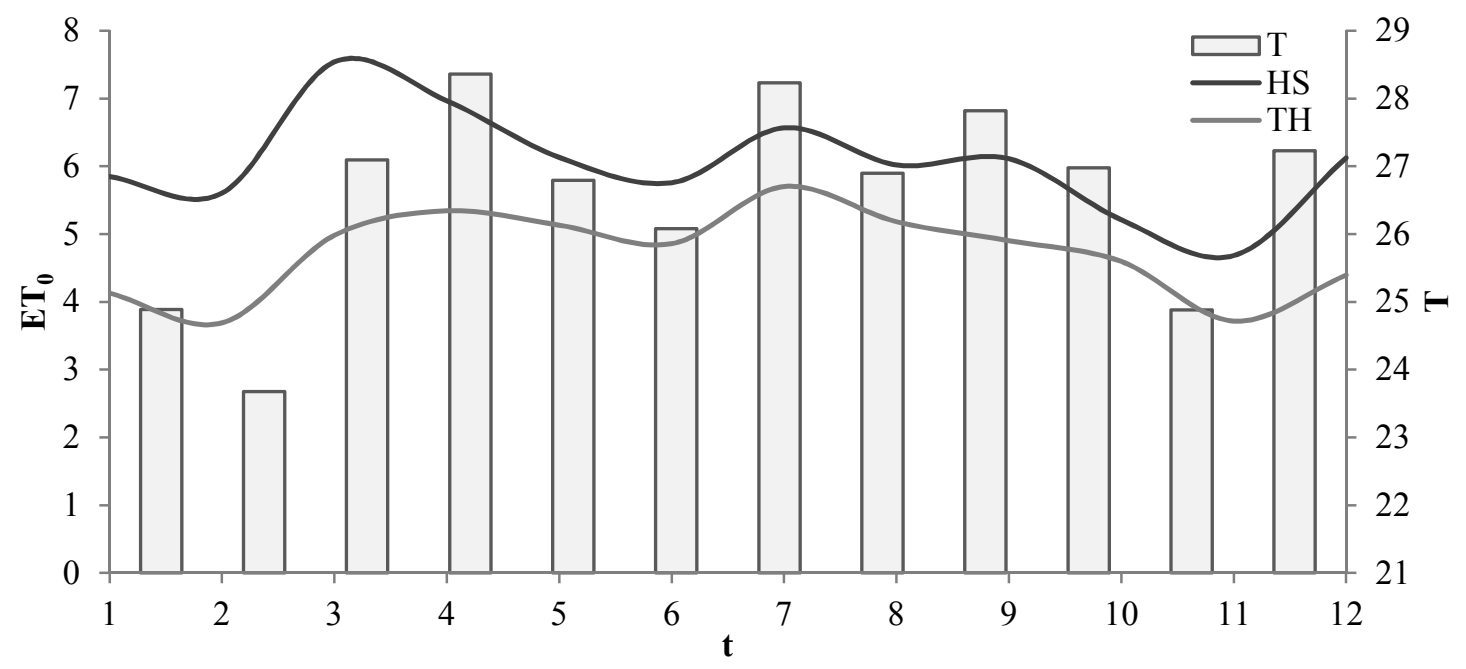

Figura 2. Valores de médias semanais da evapotranspiração de referência $\left(\mathrm{ET}_{0}, \mathrm{~mm}_{\mathrm{dia}}{ }^{-1}\right)$ estimada pelos métodos de Thornthwaite (1948) (TH) e Hargreaves \& Samani (1985) (HS), e temperatura média semanal $\left(\mathrm{T},{ }^{\circ} \mathrm{C}\right)$, ao longo do tempo $(\mathrm{t}$, semanas após o transplante). Piracicaba-SP, 2013. 
Segundo Lu et al. (2005) a diferença existente entre os métodos pode ser devida à região na qual o método foi desenvolvido.

Após estimada $\mathrm{ET}_{0}$ foi possível calcular o coeficiente de cultura (Kc). Devido à diferença entre cultivares na ETc, o Kc foi calculado para cada cultivar, utilizando $\mathrm{ET}_{0}$ estimada pelos dois métodos, Thornthwaite (1948) e Hargreaves \& Samani (1985) (Figura 3). Os valores de Kc, em geral, procederam de acordo com a ETc, apresentando seus menores valores nas primeiras semanas após o plantio (SAT) e seus maiores valores da $8^{\mathrm{a}}$ à $11^{\mathrm{a}} \mathrm{SAT}$. Para o arroz 'Batatais', os menores valores de Kc foram de 0,5 e 0,3 (2 $2^{\text {a }}$ SAT) e os maiores de 2,0 e 1,6 ( $\left.9^{\mathrm{a}} \mathrm{SAT}\right)$, para os métodos de Thornthwaite (1948) e Hargreaves \& Samani (1985), respectivamente. No arroz BRSPrimavera, os menores valores de Kc foram de 0,5 e 0,3 e os maiores de 1,9 e 1,6, para os métodos de Thornthwaite (1948) $\left(9^{\mathrm{a}}\right.$ e $10^{\mathrm{a}}$ SAT) e Hargreaves \& Samani (1985) $\left(11^{\mathrm{a}}\right.$ SAT), respectivamente.

O modelo de regressão polinomial de terceiro grau foi o que melhor se adequou à distribuição dos valores de Kc ao longo do ciclo da cultura para ambos genótipos e métodos (Figura 3: 1 e 2). O coeficiente de determinação $\left(\mathrm{R}^{2}\right)$ foi de $90,9 \%$ e $87,4 \%$ para 'Batatais' e 'BRS-Primavera', respectivamente, utilizando $\mathrm{ET}_{0}$ estimado pelo método de Thornthwaite (1948) (Figura 3: 1), e de $92,08 \%$ e $87,77 \%$ para 'Batatais' e 'BRS-Primavera', respectivamente, utilizando $\mathrm{ET}_{0}$ estimada pelo método de Hargreaves \& Samani (1985). Benli et al. (2006) também adotaram o modelo de terceiro grau que melhor se ajustou à disposição dos dados de coeficiente da cultura da alfafa.

A média dos valores observados de índice de área foliar foram: 5,9 para 'Batatais' e 4,4 para 'BRS-Primavera', em estádio R2 (47 dias após o transplante DAT); 7,4 para 'Batatais' e 5,2 para 'BRSPrimavera', em estádio R5 (66 DAT); e 7,2 para 'Batatais' e 5,2 para 'BRS-Primavera', em estádio R9 (84 DAT). Em plantas de arroz o IAF varia conforme o genótipo. Guimarães et al. (2008), avaliando cultivares com divergência fenotípica, observaram valores de IAF que variaram de 2,9 a 6 $\mathrm{m}^{2} \cdot \mathrm{m}^{-2}$. Ying et al. (1998) observaram IAF de 5,7 a $8,2 \mathrm{~m}^{2} \cdot \mathrm{m}^{-2}$, em ambiente tropical, e de 6,7 a $10,4 \quad \mathrm{~m}^{2} \cdot \mathrm{m}^{-2}$, em ambiente subtropical, para os mesmos genótipos. Vale lembrar que o cálculo do IAF é dependente da área da superfície à qual a planta está disposta, portanto, para qualquer alteração no tamanho da área da superfície do solo, há uma alteração no valor de IAF. 


\section{EVAPOTRANSPIRAÇÃO POTENCIAL E COEFICIENTE DA CULTURA DE DOIS GENÓTIPOS DE ARROZ DE TERRAS ALTAS}
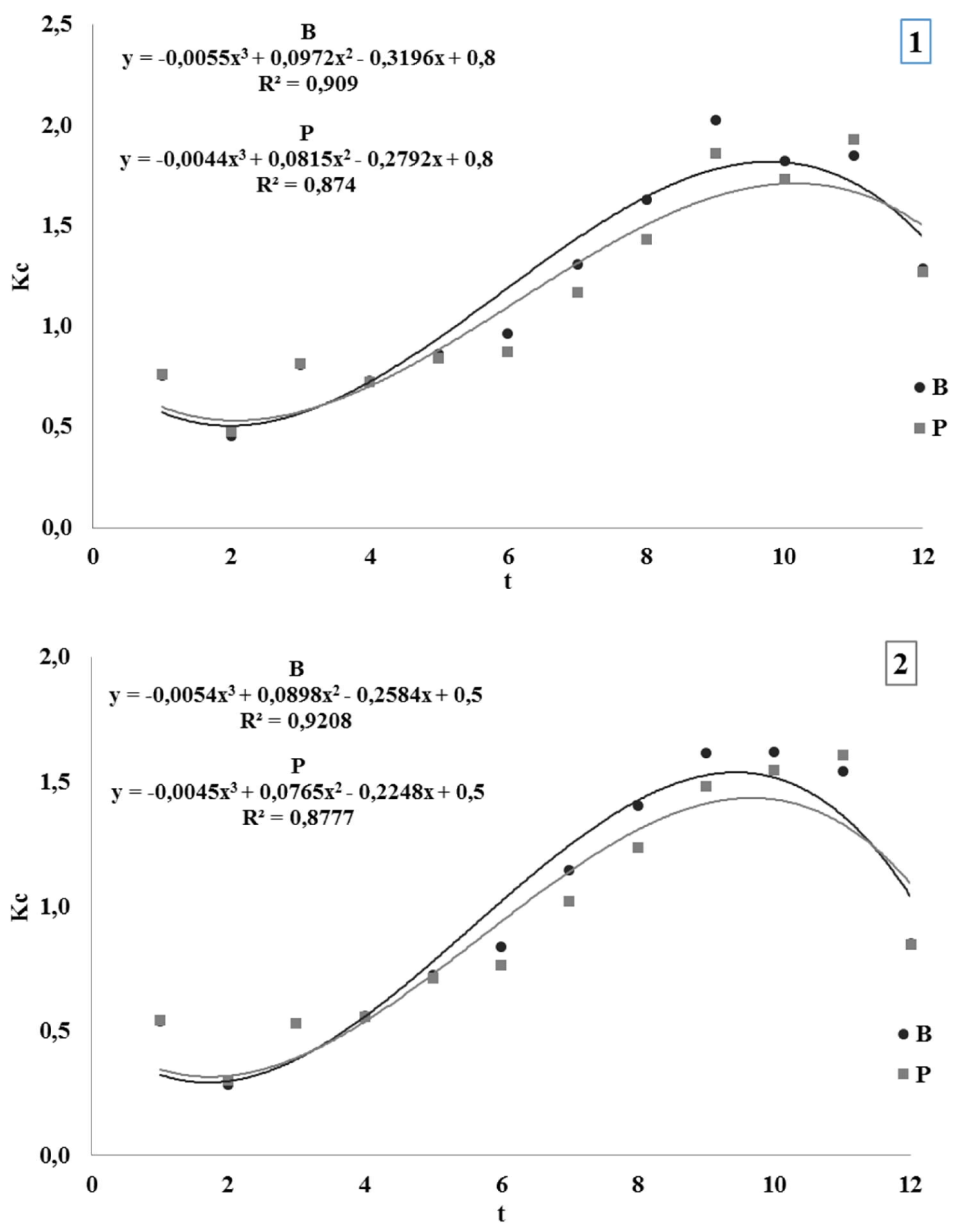

Figura 3. Valores médios semanais do coeficiente da cultura $(\mathrm{Kc})$, de cultivares de arroz de terras altas, Batatais (B) e BRS-Primavera (P), utilizando evapotranspiração de referência estimada pelos métodos de Thornthwaite (1948) (Figura 3.1), e Hargreaves \& Samani (1985) (Figura 3.2), em função do tempo (t, semanas após o transplante), Piracicaba-SP, 2013. 
Os valores observados no atual experimento são maiores que os observados por Alvarez et al. (2012), onde os máximos valores de IAF, aos 83 dias após a emergência, foram de $5,8 \mathrm{~m}^{2} \cdot \mathrm{m}^{-2}$, para $\mathrm{o}$ arroz tradicional Caiapó, e de $4,3 \mathrm{~m}^{2} \cdot \mathrm{m}^{-2}$ de terreno para o arroz BRS-Primavera. O IAF também pode variar para uma mesma variedade conforme as condições do ambiente de cultivo. Avaliando o desempenho do IAF do arroz de terras altas IAC 47 em doze experimentos, Pinheiro \& Guimarães (1990) observaram valores que variaram de 1,5 a 8,3. Analisando o crescimento e produtividade de cultivares de arroz de terras altas, Alvarez et al. (2012) constataram que a maior produtividade da cultura está relacionada mais com a fertilidade das espiguetas e massa de mil grãos, do que com o índice de área foliar. Além do mais, os autores também associaram à maior produtividade, à maior massa seca da panícula e à maior taxa de assimilação líquida.

O modelo de regressão linear foi o que melhor se adequou aos valores de $\mathrm{Kc}$ em função dos IAF estimados, tanto para o arroz Batatais e BRS-Primavera (Figura 4). Para 'Batatais' os valores de $\mathrm{R}^{2}$ foram de $77,97 \%$ e 74,37\% e para 'BRS-Primavera' foram de $85 \%$ e $85,15 \%$, utilizando como métodos de estimativa da $\mathrm{ET}_{0}$, Thornthwaite (1948) e Hargreaves \& Samani (1985), respectivamente. Stone \& Pereira (1994) também observaram relação linear entre Kc e IAF para cultivares de arroz de terras altas, com valores de $\mathrm{R}^{2}$ variando de 0,91 a 0,99 .

Adotando índice de área foliar (IAF) baixo de 0,5 no modelo de regressão linear, o arroz BRS-Primavera apresentou Kc $8,77 \%$ e $8,51 \%$ maior que o Kc do genótipo 'Batatais', utilizando os métodos de estimativa da $\mathrm{ET}_{0}$ de Thornthwaite (1948) e Hargreaves \& Samani (1985), respectivamente. Com IAF intermediário de 3,5, 'BRS-Primavera' apresentou Kc 10,38\% e $12,02 \%$ maior que o observado para 'Batatais', utilizando os métodos de Thornthwaite (1948) e Hargreaves \& Samani (1985), respectivamente. Com IAF de 6,5, 'BRS-Primavera' apresentou Kc 10,83\% e $12,79 \%$ maior que 'Batatais', utilizando os métodos de Thornthwaite (1948) e Hargreaves \& Samani (1985), respectivamente. Estes resultados demonstram que, por unidade de índice de área foliar, o arroz 'BRS-Primavera' possui maior perda de água. A quantidade de água perdida pela planta para a atmosfera está relacionada com a densidade, tamanho, posição, abertura e fechamento dos 
estômatos (HETHERINGTON \& Indica IR72 e a variedade Japonica tropical WOODWARD, 2003).

IR69093-41-3-2-2, Laza et al. (2010)

As características dos estômatos observaram que a variedade Indica podem estar relacionadas com a origem da aumentou a densidade de estômatos e a cultivar. Em estudo com linhagens variedade Japonica tropical aumentou o resultantes do cruzamento entre a variedade tamanho dos estômatos nos seus derivados.

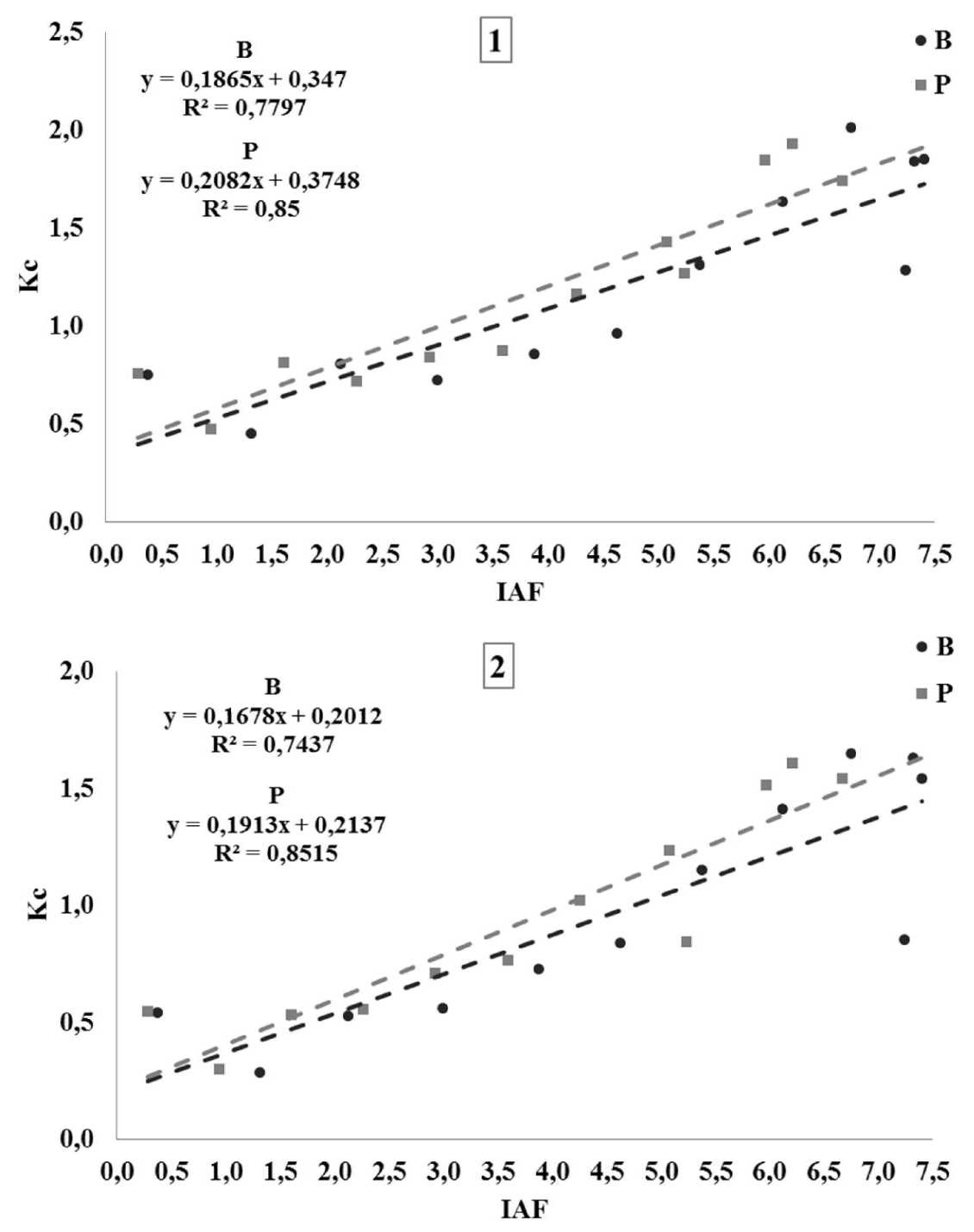

Figura 4 Valores de médias semanais do coeficiente da cultura ( $\mathrm{Kc}$ ) calculado usando evapotranspiração de referência estimada pelos métodos de Thornthwaite (1948) (Figura 4.1) e Hargreaves \& Samani (1985) (Figura 4.2), de cultivares de arroz de terras altas, Batatais (B) e BRS-Primavera (P), em função dos valores médios semanais de índice de área foliar estimados $\left(\mathrm{IAF}_{\mathrm{est}}\right)$. Piracicaba-SP, 2013. 
Para Japonica tropical, os autores observaram que a densidade estomática foi de 579 e 653 estômatos. $\mathrm{mm}^{-2}$, e tamanho médio de 23,2 e 22,3 $\mu \mathrm{m}$, para a superfície adaxial e abaxial da folha respectivamente, e para Indica, 635 e 750 estômatos. $\mathrm{mm}^{-2}$ e tamanho médio de 22,2 e 21,2 $\mu \mathrm{m}$. Comparando com outras espécies anuais, folhas de arroz possuem maior número de estômatos por área foliar que folhas de milho, que apresentam em torno de 70 e 100 estômatos. $\mathrm{mm}^{-2}$ (DRISCOLL et al., 2006), que folhas de trigo, que possuem cerca de 85 e 60 estômatos. $\mathrm{mm}^{-2}$ (MEHRI et al., 2009) e, que folhas de soja, em torno de 100 e 220 estômatos.mm ${ }^{-2}$ (BUTTERY et al., 1993). Quanto à posição dos estômatos, a quantidade em folhas de arroz é similar tanto na superfície adaxial quanto na abaxial (OHSUMI et al., 2007). No entanto, a densidade de estômatos responde a fatores ambientais (ISHIMARU et al., 2001).

Em estudos com concentrações de $\mathrm{CO}_{2}$ no ambiente, Rowland-Bamford et al. (1990) constataram que em concentração de $\mathrm{CO}_{2}$ de $330 \mu 1 . \mathrm{L}^{-1}$, a densidade encontravase em torno de 300 e 341 estômatos. $\mathrm{mm}^{-2}$ nas superfícies abaxial e adaxial da folha, respectivamente, porém, em concentração alta de $\mathrm{CO}_{2}\left(900 \mu 1 . \mathrm{L}^{-1}\right)$, a quantidade de estômatos aumentou para próximo a 490 e
440 estômatos. $\mathrm{mm}^{-2}$. Portanto, plantas que são conduzidas em condições sem limitações hídricas podem apresentar maior densidade estomática que plantas que evoluíram em ambientes com instabilidade climática.

A menor perda de água por área de folha do arroz Batatais pode ser devido este ser material oriundo de coletas realizadas em locais onde seu cultivo é realizado há diversos anos, passando por diversas variações climáticas e adquirindo tolerância de forma natural. Enquanto o arroz BRSPrimavera é material desenvolvido por programa de melhoramento genético, visando prioritariamente a produtividade e qualidade de grãos, sem passar por condições extremas do ambiente e sem limitação hídrica.

\section{CONCLUSÕES}

O genótipo de arroz de terras altas Batatais apresentou na $8^{\mathrm{a}}$ semana após o plantio, maior evapotranspiração, que o genótipo BRS-Primavera.

Conforme a relação do coeficiente da cultura e o índice de área foliar estimado, o genótipo BRS-Primavera apresentou maior evapotranspiração por índice de área foliar. 


\section{REFERÊNCIAS BIBLIOGRÁFICAS}

ABDULLAHI, A.S.; SOOM, M.A.M.; AHMAD, D.; SHARIFF, A.R.M. 2013.Characterization of rice (Oryza sativa) evapotranspiration using micro paddy lysimeter and class "A" pan in tropical environments. Australian Journal of Crop Science, Melbourne, v. 7, n. 5, p. 650-658.

ALVAREZ, R.C.F.; CRUSCIOL, C.A.C.; NASCENTE, A.S. 2012. Análise de crescimento e produtividade de cultivares de arroz de terras altas dos tipos tradicional, intermediário e moderno. Pesquisa Agropecuária Tropical, Goiânia, v. 42, n. 4, p. 397406, out./dez.

ANA - Agência nacional da água. 2009. Disponível em:< http://cadastrocthidro.ana.gov.br/relatori oProjeto.php?\&id $=637 \&$ voltar $=$ projetos \&orderBy $=\&$ filtro $=\&$ regs $=10 \& b \mathrm{~b}=\& b n=$ $\&$ pag=1>. Acesso em: 20 Abr. 2009.

BENLI, B.; KODAL, S.; ILBEYI, A.; USTUN, H. 2006. Determination of evapotranspiration and basal crop coefficient of alfalfa with a weighing lysimeter. Agricultural Water Management, Amsterdam, v. 81, p. 358-370.

BUTTERY, B.R.; TAN, C.S.; BUZZELL, R.I.; GAYNOR, J.D.; MACTAVISH, D.C. 1993. Stomatal numbers of soybean and response to water stress. Plant and Soil, Crawley, v. 149, p. 283-288.

CHAN, C.S.; CHEONG, A.W. 2001. Seasonal weather effects on crop evapotranspiration and rice yield. Journal of Tropical Agriculture and Food Science, Kuala Lumpur, v. 29, n. 1, p. 77-92.

DATTA, S.K. 1981. Principles and practices of rice production. A WileyIntersciences publication. 637p.

DRISCOLL, S.P; PRINS, A.; OLMOS, E.; KUNERT, K.J.; FOYER, C.H. 2006.
Specification of adaxial and abaxial stomata, epidermal structure and photosynthesis to $\mathrm{CO}^{2}$ enrichment in maize leaves. Journal of Experimental Botany, Oxford, v. 57, n. 2, p. 381-390.

DUCHEMIN, B.; HADRIA, R.; ERRAKI, S.; BOULET, G.; MAISONGRANDE, P.; CHEHBOUNI, A.; ESCADAFAL, R.; EZZAHAR, J.; HOEDJES, J.C.B.; KHARROU, M.H.; KHABBA, S.; MOUGENOT, B.; OLIOSO, A.; RODRIGUEZ, J.-C.; SIMONNEAUX, V. 2006. Monitoring wheat phenology and irrigation in Central Morocco: On the use of relationships between evapotranspiration, crops coefficients, leaf area index and remotely-sensed vegetation indices. Agricultural Water Management, Amsterdam, v. 79, p. 127.

FAOSTAT - Food and Agriculture Organization - FAO. 1991. Disponível em: <www.fao.org.br>. Acesso em: 10 Abr. 2012.

FORNASIERI FILHO, D.; FORNASIERI, J.L. 2006. Manual da cultura do arroz. Jaboticabal: FUNEP.589p.

GUIMARÃES, C.M.; SANTOS, A.B.; MAGALHÃES JÚNIOR, A.M.; STONE, L.F. 2006. Sistemas de cultivo. In: SANTOS, A.B. dos; STONE, L.F.; VIEIRA, N.R. de A. A Cultura do Arroz no Brasil. 2. ed. rev. ampl., Santo Antônio de Goiás: Embrapa Arroz e Feijão. p. 257-288.

GUIMARÃES， C.M.; STONE， L.F.; NEVES, P.C.F. 2008. Eficiência produtiva de cultivares de arroz com divergência fenotípica. Revista Brasileira de Engenharia Agrícola e Ambiental, Campina Grande, PB, v. 12, n. 5, p. 465-470.

HARGREAVES G.H. SAMANI, Z.A. 1985. Reference crop evapotranspiration from temperature. Applied Engineering in Agriculture, Saint Joseph, v. 1, n. 2, p. 96-99. 
HEINEMANN, A.B.; STONE, L.F. 2009. Efeito da deficiência hídrica no desenvolvimento e rendimento de quatro cultivares de arroz de terras altas. Pesquisa Agropecuária Tropical, Goiânia, v. 39, n. 2, p. 134-139, abr./jun.

HETHERINGTON, A.M.; WOODWARD, F.I. 2003. The role of stomata in sensing and driving environmental change.

Nature, London, v. 424, p. 901-908.

IBGE - INSTITUTO BRASILEIRO DE GEOGRAFIA E ESTATÍSTICA. 2014. Levantamento Sistemático da Produção Agrícola. Rio de Janeiro, v.27, n.1, p.1-85.

ISHIMARU, K.; SHIROTA, K.; HIGA, M.; KAWAMITSU, Y. 2001. Identification of quantitative trait loci for adaxial and abaxial stomatal frequencies in Oryza sativa. Plant Physiology and Biochemistry, Stuttgart, v. 39, n. 2, p. 173-177.

LAZA, MA. R.C.; KONDO, M.; IDETA, O.; BARLAAN, E.; IMBE, T.2010. Quantitative trait loci for stomatal density and size in lowland rice. Euphytica, Wageningen, v. 172, n. 2, p. 149-158.

LILLEY, J.M.; FUKAI, S. 1994. Effect of timing and severity of water deficit on four diverse rice cultivars. IIPhysiological responses to soil water deficit. Field Crops Research, Amsterdam, v. 37, p. 215-223.

LU, J.; SUN, G.; MCNULTY, S.G.; AMATYA, D.M. 2005. A comparison of six potential evapotranspiration methods for regional use in the South Eastern United States. Journal of the American Water Resources Association, Washington, v. 41, n. 3, p. 621-633.

MEHRI, N.; FOTOVAT, R.; SABA, J.; JABBARI, F. 2009. Variation of stomata dimensions and densities in tolerant and susceptible wheat cultivars under drought stress. Journal of Food,
Agriculture \& Environment, Helsinki, v. 7, n.1, p.167-170.

OHSUMI, A.; KANEMURA, T.; HOMMA, K.; HORIE, T.; SHIRAIWA, T. 2007. Genotypic variation of stomatal conductance in relation to stomatal density and length in rice (Oryza sativa L.) Plant Production Science, Tokyo, v. 10, n. 3, p. 322-328.

PINHEIRO, B. da S.; GUIMARÃES, E. P. 1990. Índice de área foliar e produtividade do arroz de sequeiro. 1. Níveis limitantes. Pesquisa Agropecuária Brasileira, Brasília, v. 25, n. 6, p. 863-872.

PRAVEEN, P.; DEVARANAVADGI, V.S.; DESAI, G.A.; PRAVEEN, G.S.; BAI, K.P. M. 2012. Estimation of evapotranspiration rate by different methods for paddy crop in South Kodagu, Central Western Ghats. International Journal of Agricultural Engineering, Uttar Pradesh, v. 5, i. 1, p. 69-72, april.

ROWLAND - BAMFORD, A.J.; NORDENBROCK, C.; BAKER, J.T.; BOWES, G.; ALLEN, L.H. 1990. Changes in stomatal density in rice grown under various $\mathrm{CO}_{2}$ regimes with natural solar irradiance. Environmental and Experimental Botany, Elmsford, v.2, p.175-180.

THORNTHWAITE, C.W. 1948. An approach toward a rational classification of climate. Geographical Review, New York, v.38, n. 1, p.55-94.

YING, J.; PENG, S.; HE, Q.; YANG, H.; YANG, C.; VISPERAS, R. M.; CASSMAN, K. G. 1998. Comparison of high-yield rice in tropical and subtropical environments I. determinants of grain and dry matter yields. Field Crops Research, Amsterdam, v. 57, p. 71-84.

Recebido em: 30/11/2014 Aceito para publicação em: 14/9/2015 